\title{
INVESTIGAÇÃO DO CONHECIMENTO DE PACIENTES SUBMETIDOS À HEMODIÁLISE SOBRE A FINALIDADE DO USO DE SUPLEMENTOS EM SEU TRATAMENTO
}

\author{
Isabella S. Abreu ${ }^{1}$, Taísa H. Pereira ${ }^{2}$
}

RESUMO: Estudo quantitativo realizado na Clínica de Doenças Renais Ltda no município de Guarapuava-PR. Objetivos: caracterizar os pacientes em tratamento hemodialítico segundo algumas variáveis sócio-demográficas e clínicas; avaliar seu conhecimento sobre a finalidade do uso dos suplementos utilizados no tratamento. Coletou-se os dados mediante um questionário com questões abertas e fechadas, durante as sessões de hemodiálise. Em relação à etiologia, houve um predomínio da Glomerulonefrite Crônica (46\%), quanto ao uso de suplementos $100 \%$ dos pacientes recebiam a eritropoetina recombinante humana, 54,5\% faziam uso do carbonato de cálcio, 54,5\% utilizavam o ferro injetável e $9 \%$ a vitamina D. A maior parte dos entrevistados souberam relatar qual suplemento utiliza e qual a sua finalidade; enquanto que a maioria dos pacientes não sabia relatar, quando era administrado e com que periodicidade eram ingeridos estes suplementos e também não sabiam referir porque deviam ser tomados com rigidez de horário conforme prescrito pelo médico. Conclui-se com os dados obtidos a grande importância da orientação clara e adequada de todos os pacientes em programa de hemodiálise, que fazem uso de suplementação, pela equipe multiprofissional : enfermagem, médica e de nutrição.

PALAVRAS-CHAVE: Suplementos; Hemodiálise; Insuficiência renal crônica.

\section{INVESTIGATION ONTHE KNOWLEDGE OF PATIENTS UNDERGOING HEMODIALYSIS ABOUT THE PURPOSE IN THE USE OF SUPPLEMENTS IN THEIR TREATMENT}

ABSTRACT: It's a quantitative study carried out at the Clinic of Renal Diseases Ltd. in the municipality of Guarapuava/ Paraná State - Brazil. Objectives: to characterize patients undergoing hemodialysis according to some social-demographic and clinical variables; to assess their knowledge on the purpose of the use of some nutritional supplements during their treatment. Data were collected through a questionnaire of open and close questions during hemodialysis sessions. Regarding etiology, chronic glomerulonephritis was prevalent ( $46 \%$ ); concerning the use of supplements, all patients ( $100 \%$ ) were receiving recombinant human erythropoietin, $54.5 \%$ made use of calcium carbonate, $54.5 \%$ were administered parenteral iron and $9 \%$ vitamin $\mathrm{D}$. The majority of the interviewed patients were able to tell what supplement they were receiving and what it was for, although most of them didn't know when the supplements were administered or how often they were taken, or why they should be taken at prescribed hours. With the obtained data, it can be concluded that clear and adequate guidance should be provided by the multiprofessional team ( nurses, physicians, nutritionists ) to patients in hemodialysis programs and taking supplements.

KEYWORDS: Supplements; Hemodialysis; Chronic renal condition.

\section{INVESTIGACIÓN SOBRE EL CONOCIMIENTO DE LOS PACIENTES SOMETIDOS AL TRATAMIENTO HEMODIALÍTICO ACERCA DE LA FINALIDAD DEL USO DE SUPLEMENTOS EM SU TRATAMIENTO}

RESUMEN: Estudio cuantitativo con los objetivos de caracterizar los pacientes en tratamiento hemodialítico según algunas variables sociodemograficas y clínicas y evaluar su conocimiento sobre la finalidad del uso de los suplementos utilizados en el tratamiento. La colecta de los datos fué realizada por medio de un cuestionário con preguntas abiertas y cerradas, durante las sesiones de hemodiálisis. El local de la colecta de los datos fué la Clínica de Doenças Renais en el municipio de Guarapuava, PR. En relación a la etiología, hubo predominio de la Glomerulonefrite Crónica (46\%), cuanto al uso de suplementos, $100 \%$ de los pacientes recebian eritropoetina recombinante humana, 54,5\% hacían uso do carbonato de cálcio, 54,5\% utilizavan hierro injetable y 9\%, a vitamina D. La gran parte de los entrevistados supo decir cual suplemento usa y su finalidad, pero no supo decir cuando era administrado y con que frecuencia eran tomados estes suplementos y tambien no sabian decir por que devian ser tomados con riguracidad de horários segun la prescrición médica. Se concluye con los datos obtenidos la gran importancia de la orientación clara y adecuada de todos los pacientes en programa de hemodiálise, que hacen uso de suplementación, por la equipe multiprofesional: enfermería, médica y de nutrición.

PALAVRAS CLAVES: Suplementos, Hemodiálisis, Insuficiencia renal crónica.

\footnotetext{
${ }^{1}$ Enfermeira. Mestre em Saúde Pública. Departamento de Enfermagem da Universidade Estadual do Centro-Oeste-UNICENTRO. ${ }^{2}$ Nutricionista. Especialista em Nutrição Clínica. CLIRE-Clínica de Doenças Renais Ltda.
}

Autor correspondente:

Isabella S. Abreu

Rua Brigadeiro Rocha, 1141 - 85010-210 - Guarapuava-PR

Recebido: 25/03/08

E-mail:i_enf@yahoo.com.br

Aprovado: 05/07/08

Cogitare Enferm 2008 Jul/Set; 13(3):422-7 


\section{INTRODUÇÃO}

A Insuficiência Renal Crônica (IRC) refere-se a um diagnóstico sindrômico de perda progressiva e, geralmente irreversível, da função renal de depuração, ou seja, da filtração glomerular ${ }^{(1)}$, que é parcialmente substituída por diferentes modalidades de tratamento, entre os quais a hemodiálise.

Os maiores determinantes da morbidade e mortalidade em hemodiálise são o estado nutricional dos pacientes e a adequacidade da diálise. Provavelmente existe uma inter-relação significativa entre esses dois fatores, pois os pacientes bem dialisados apresentam maior bem estar geral e, conseqüentemente, melhor ingestão alimentar ${ }^{(2)}$.

A desnutrição calórico-protéica é comum nos pacientes em hemodiálise, de causa multifatorial e inclui variáveis associadas à ingestão alimentar deficiente, distúrbios hormonais e gastrointestinais, restrições rigorosas na dieta, uso de medicamentos que podem influenciar na absorção de nutrientes, diálise insuficiente e presença de enfermidades intercorrentes.

Em hemodiálise as recomendações de vários nutrientes são restritivas, entretanto, a desnutrição pode ser um problema maior para a morbimortalidade do que o risco imposto por uma dieta mais liberal ${ }^{(2)}$.

Devido às deficiências impostas pela IRC, durante o tratamento também se faz necessário o uso de suplementação medicamentosa indicada conforme a necessidade de cada paciente e avaliada mensalmente por meio de exames laboratoriais. Os principais suplementos utilizados pelos pacientes da CLIRE - Clínica de Doenças Renais Ltda são: o Ferro, a Eritropoetina, o Carbonato de Cálcio, a Vitamina D e a Vitamina C.

Durante seu tratamento e a cada sessão de diálise, o paciente é submetido ao uso dos suplementos acima citados, porém muitas vezes não conhece a finalidade do seu uso; desconhecimento tal que pode surgir por dificuldades relacionadas ao próprio paciente, como também por falta de orientação da equipe multidisciplinar, favorecendo a sua não adesão ao tratamento.

Os pacientes renais crônicos se tornando desanimados e sem esperança e, por estas razões ou por falta de orientação, acabam abandonando o tratamento ou não atribuindo a este a devida importância. É necessário estimular suas capacidades, para se adaptarem de maneira positiva ao novo estilo de vida e assumirem o controle de seu tratamento ${ }^{(3)}$.
Este estudo foi motivado para conhecer as principais dúvidas e dificuldades dos pacientes em relação ao uso da suplementação medicamentosa, visando ações educativas e melhoria da assistência e da qualidade de vida destes pacientes. Acreditando que a essência fundamental da enfermagem é o cuidado, também pretendemos com esse estudo caracterizar algumas variáveis sócio-demográficos destes pacientes, a fim de enriquecer os dados sistematizados, os quais poderão subsidiar o planejamento dos serviços ofertados e visando a qualidade deste cuidado.

\section{OBJETIVOS}

Caracterizar os pacientes em tratamento hemodialítico segundo algumas variáveis sóciodemográficas e clínicas.

Avaliar o conhecimento de pacientes em hemodiálise sobre a finalidade do uso de suplementos em seu tratamento.

\section{METODOLOGIA}

Trata-se de uma pesquisa descritiva de caráter quantitativo. Fizeram parte deste estudo 11 pacientes da CLIRE no município de Guarapuava - PR, em programa regular de hemodiálise três vezes por semana e no período vespertino. O estudo foi realizado no mês de julho de 2002; para a coleta dos dados foi utilizado um instrumento contendo questões abertas e fechadas, aplicado pela pesquisadora e referentes aos dados sócio-demográficos, clínicos, sobre os suplementos utilizados pelos pacientes durante o tratamento e o conhecimento acerca destes suplementos.

A análise dos dados foi realizada por meio de estatística descritiva e discutida à luz da literatura especializada. A realização deste estudo atendeu a resolução 196/96 do Conselho Nacional de Saúde, que estabelece as diretrizes e normas regulamentadoras de pesquisas envolvendo seres humanos, respeitando o sigilo e anonimato das informações e com o consentimento da direção clínica da CLIRE.

\section{RESULTADOS}

Entre os 99 pacientes em hemodiálise atendidos na CLIRE no período estudado, 20 realizam hemodiálise no período vespertino. Destes, 11 (55\%) participaram da pesquisa, 5 (25\%) foram excluídos por apresentarem instabilidade clinica ou dificuldade 
de compreensão e 4 (20\%) recusaram-se a participar.

\section{Variáveis sócio-demográficas}

\section{Sexo}

Nesta variável, 54,5\% (n=6) eram do sexo masculino e 45,5\% (n=5) do sexo feminino. Em estudo realizado por Castro et $\mathrm{al}^{(4)}$, sobre Qualidade de Vida de pacientes com IRC em hemodiálise, dos 184 pacientes estudados $63 \%$ eram do sexo masculino. $\mathrm{O}$ sexo masculino foi encontrado em maior percentual em ambos os estudos.

\section{Etiologia da Insuficiência Renal Crônica (IRC)}

A IRC pode ser causada por doenças sistêmicas como diabetes mellitus; glomerulonefrite crônica; pielonefrite; hipertensão não controlada; obstrução do trato urinário; lesões hereditárias (doença renal policística); distúrbios vasculares; infecções; medicamentos; agentes tóxicos; agentes ambientais e ocupacionais (chumbo, cádmio, mercúrio e cromo) ${ }^{(5-7)}$.

As causas da IRC vão desde as doenças primárias dos rins, às doenças sistêmicas que acometem os rins e as doenças do trato urinário, a nefropatia diabética, hipertensão e a glomerulonefrite ${ }^{(8-9)}$. Estes dados foram também encontrados neste estudo, sendo que $46 \% \quad(n=5)$ dos pacientes apresentavam como etiologia da doença a Glomerulonefrite Crônica, 27\% $(\mathrm{n}=3)$ Nefroesclerose Hipertensiva, 18\% $(\mathrm{n}=2)$ Nefropatia Diabética e 9\% (n=1) Tuberculose Renal.

\section{Escolaridade}

Quanto à escolaridade, 72,7\% $(\mathrm{n}=8)$ tinham 0 ensino fundamental incompleto, $9,1 \%(n=1)$ o ensino fundamental completo, 9,1\% $(n=1)$ o ensino médio incompleto e $9,1 \%(\mathrm{n}=1)$ o ensino médio completo (Gráfico 1). Observa-se que a maioria dos pacientes apresentou baixo nível de escolaridade, relevante por ser um fator que interfere no grau de entendimento e compreensão dos pacientes acerca das informações e orientações recebidas sobre o seu tratamento.

Em estudo realizado sobre Qualidade de Vida de pessoas dependentes de hemodiálise, considerando alguns aspectos físicos, sociais e emocionais, $11.8 \%$ nunca estudaram e não sabem ler nem escrever e $29,4 \%$ tinham o ensino fundamental incompleto ${ }^{(10)}$. Estes dados indicam que os profissionais de saúde devem atentar à comunicação adequada utilizando linguagem acessível e de fácil entendimento, principalmente no que se refere às orientações sobre o tratamento. Os dados acima descritos estão representados no Gráfico 1.

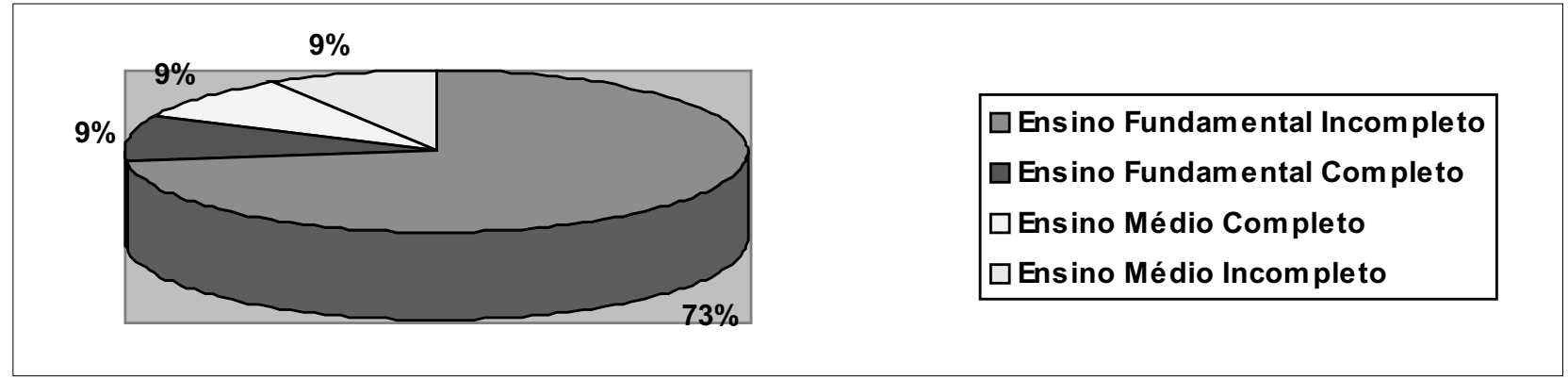

Gráfico1 - Distribuição dos pacientes (n=11) segundo a escolaridade. Guarapuava, 2002

\section{Suplementos utilizados no tratamento da IRC}
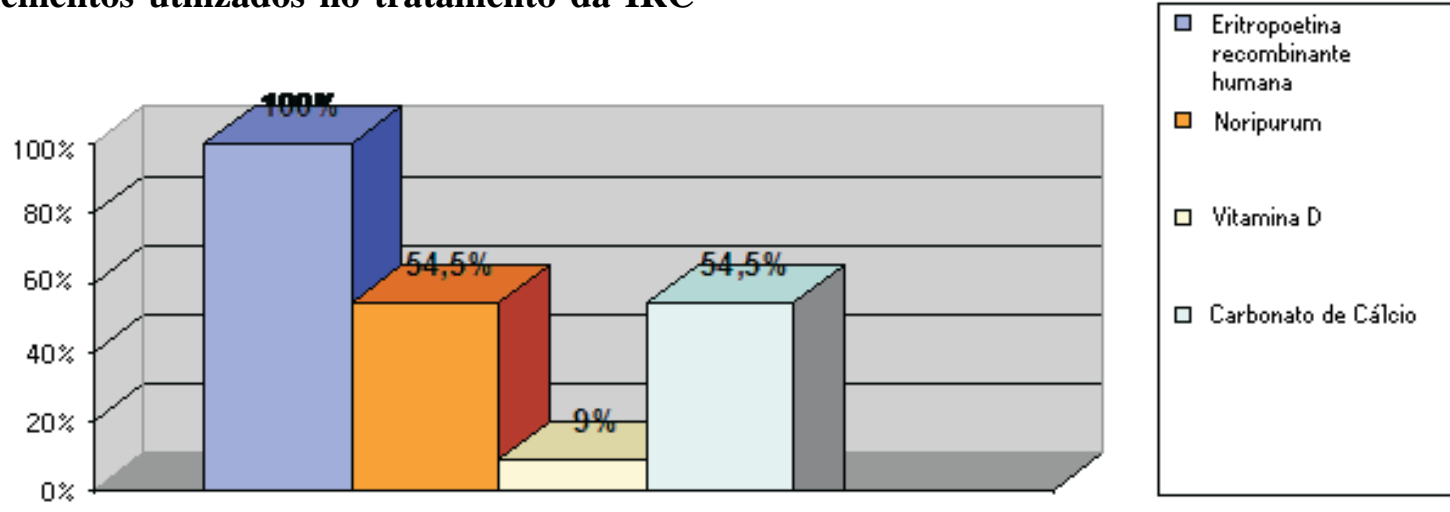

Gráfico 2 - Distribuição dos pacientes (n=11) segundo os suplementos utilizados no tratamento. Guarapuava, 2002 
No grupo estudado ( $\mathrm{n}=11), 100 \%$ utilizavam a Eritropoetina Recombinante Humana (EPO), 54,5\% faziam uso do Noripurum ${ }^{\circledR}$ (EV), 54,5\% usavam o Carbonato de Cálcio e 9\% utilizavam a vitamina D (Gráfico 2).

O conhecimento acerca dos suplementos foi diversificado, e estão abaixo apresentados.

\section{Finalidade do uso de Eritropoetina}

Do total de pacientes, quando questionados sobre o porquê do uso da Eritropoetina, 36,4\% (n=4) responderam que a finalidade é para não ter anemia, $18,2 \%(\mathrm{n}=2)$ que era para aumentar as células do sangue e 9,1\% (n=1) para "não ficar fraco". Porém, 36,4\% ( $\mathrm{n}=4)$ não sabiam responder (Gráfico 3).

A anemia, principal conseqüência hematológica da IRC, constitui o fator responsável pela limitação da capacidade física dos pacientes renais crônicos, prejudica a sua reabilitação social e profissional e acarreta uma qualidade de vida insatisfatória ${ }^{(2)}$. O principal mecanismo da anemia renal é a deficiência de eritropoetina, um hormônio glicoprotéico, produzido pelo rim em células localizadas na córtex renal e medula externa e cuja principal função é estimular a hematopoiese ${ }^{(2)}$.

Na última década, a eritropoetina recombinante humana (ERHu) conquistou seu lugar como principal terapêutica na correção da anemia, revolucionando o cuidado do paciente renal crônico. Durante este período a droga tornou-se largamente utilizada e mostrou-se eficaz em 95\% dos pacientes tratados. O momento ideal para o início do tratamento precisa ser baseado em critérios clínicos e não somente em valores prefixados para hematócrito ou hemoglobina. Hoje é consenso que doses iniciais de $50-100 \mathrm{U} / \mathrm{Kg}$ de peso, duas a três vezes por semana, são suficientes para a maioria dos pacientes adultos em tratamento dialítico. As doses devem ser ajustadas individualmente na fase inicial de correção da anemia até atingir a hemoglobina objetivada, e podem ser reduzidas em até $30 \%$ na fase de manutenção ${ }^{(11)}$.
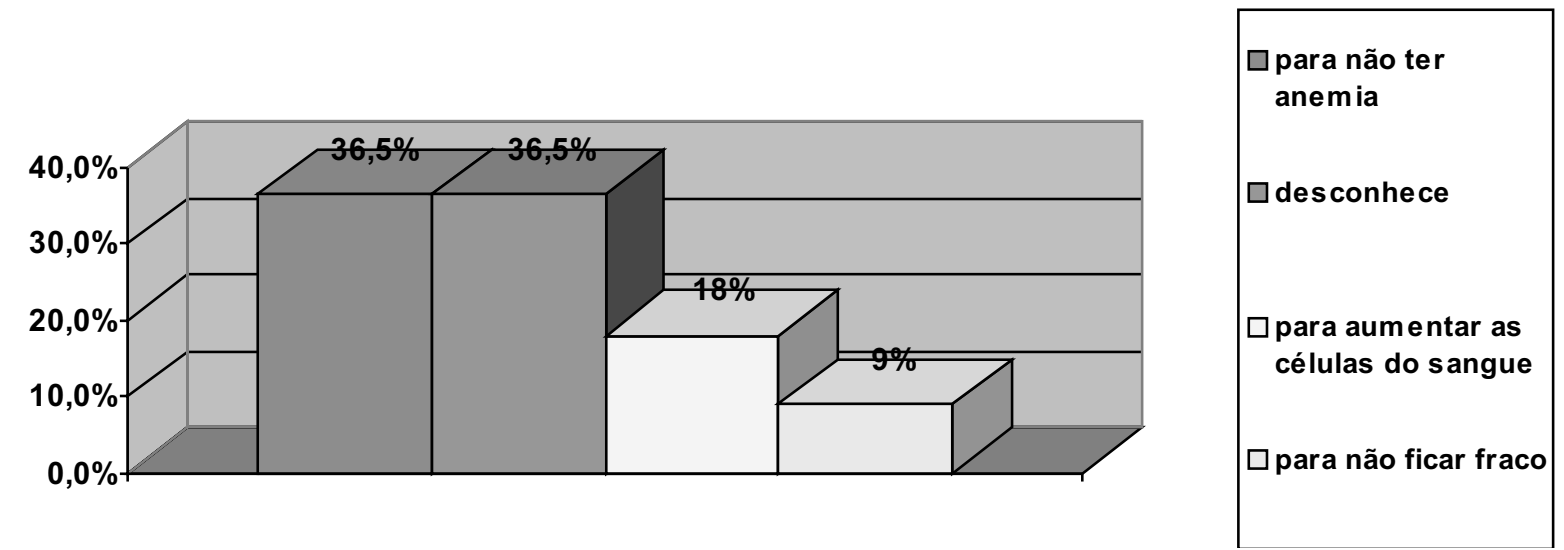

Gráfico 3 - Distribuição dos pacientes (n=11) segundo o conhecimento sobre o uso de eritropoetina. Guarapuava, 2002

\section{Finalidade do uso de Carbonato de Cálcio}

Quando questionados sobre o uso do Carbonato de Cálcio, 63,6\% (n=7) responderam que atua sobre os ossos, $18,2 \%(n=2)$ responderam ser para o funcionamento intestinal e $18,2 \%(n=2)$ para prurido (Gráfico 4).

Com a progressão da doença ocorrerá uma retenção de fósforo na circulação, havendo necessidade de se controlar sua ingestão diária. $\mathrm{O}$ monitoramento do acúmulo de fósforo na IRC é crucial na prevenção do hiperparatireoidismo secundário e de calcificações metastáticas. O tratamento da hiperfosfatemia inclui intervenções alimentares, uso de quelantes (carbonato de cálcio) e a remoção de fósforo pelo tratamento dialítico ${ }^{(2)}$.
Os quelantes de fósforo que contêm cálcio devem ser utilizados, inicialmente, em todos os pacientes. Também servem como fonte de cálcio suplementar, o que pode prevenir o aparecimento precoce das osteodistrofias, que são conseqüências da IRC. Este suplemento deve ser ingerido durante ou logo após as refeições em proporções iguais às de fósforo ingerido, para aumentar o grau de ligação do $\mathrm{Pi}^{(12)}$.

Em estudo sobre o uso de carbonato de cálcio em pacientes em diálise, mostrou que esse composto é efetivo no controle do fósforo na maioria dos pacientes, ocorrendo hipercalcemia naqueles que apresentavam alta ingestão de fósforo e, consequentemente, com o uso de maiores doses do quelante ${ }^{(13)}$. 
Finalidade do uso de Carbonato de Cálcio

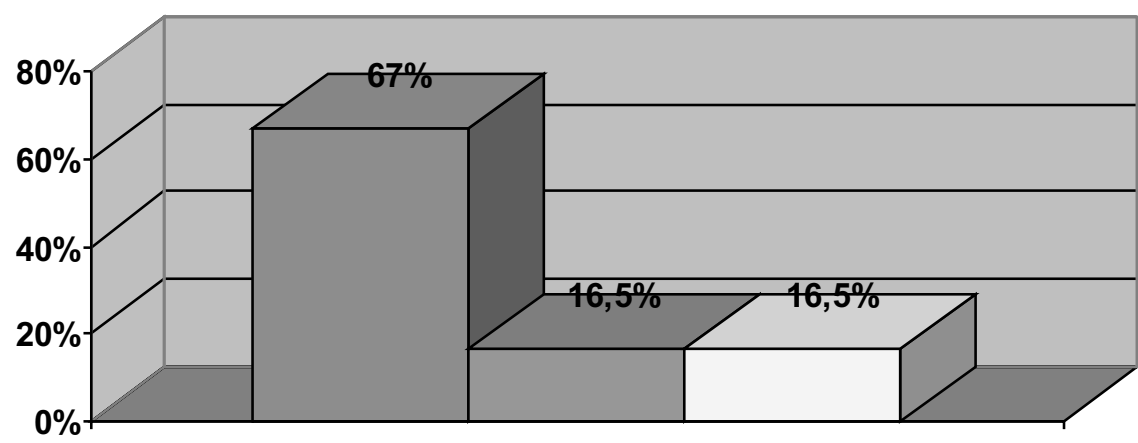

$\square$ para os ossos

$\square$ para o intestino

$\square$ para coceira

Gráfico 4 - Distribuição dos pacientes (n=11) segundo conhecimento sobre o uso de carbonato de cálcio. Guarapuava, 2002

\section{Finalidade do uso de Vitamina D}

Dos pacientes estudados, apenas um era usuário de vitamina $\mathrm{D}$ e sobre a finalidade deste suplemento, respondeu que é para fortalecimento dos ossos.

A vitamina $\mathrm{D}$ é formada tanto a partir de fontes alimentares, como da síntese endógena que ocorre na pele. Os principais órgãos afetados são o intestino, sua ação resulta em aumento da absorção de cálcio, e nos ossos onde apresenta duas ações distintas: maior mobilização de cálcio do osso, pelo aumento da atividade e do número de osteoclastos, e facilita a mineralização óssea, provavelmente por suprir quantidades adequadas de cálcio para a matriz óssea ${ }^{(2)}$.

\section{Finalidade do uso de Ferro}

Quanto a finalidade da suplementação com ferro, $45,4 \%(n=5)$ respondeu ser para anemia, 18,2\% $(n=2)$ desconhecem sua finalidade, $18,2 \%(\mathrm{n}=2)$ referiram estarem utilizando este suplemento porque estavam com o "ferro baixo" e $18,2 \%(n=2)$ referiram que é para controlar a falta de ferro (Gráfico 5). Pelos dados encontrados podemos observar que a maioria dos pacientes conhece a indicação e por que fazem uso deste suplemento, visto que seu uso é recomendado no diagnóstico da anemia, comum em pacientes com IRC.

A anemia está presente na maioria dos pacientes renais crônicos. Sabe-se que a causa mais comum é a produção deficiente do hormônio eritropoietina. Porém, supõe-se que as toxinas urêmicas também inibam a eritropoiese e reduzam o tempo de vida dos eritrócitos. Normalmente é prescrito um suplemento de ferro para pacientes em hemodiálise ou diálise peritoneal contínua. Existem, porém, controvérsias sobre qual é o melhor método para a sua suplementação, sendo três vias utilizadas: endovenosa, oral e intramuscular. Recomendase que para adultos, sejam administrados $100 \mathrm{mg}$ de ferro endovenoso a cada sessão de hemodiálise, em 10 doses, ou $100 \mathrm{mg}$ semanais durante 10 semanas $^{(2)}$.

Após duas semanas do término da terapia, são avaliados o hematócrito, a hemoglobina, a ferritina sérica e a porcentagem de saturação de transferrina. Pensa-se que, uma vez alcançados os níveis ótimos de hematócrito, hemoglobina e das reservas de ferro, seja necessária uma dose endovenosa de manutenção, variando de 25 a $100 \mathrm{mg}$ por semana para os pacientes em hemodiálise ${ }^{(2)}$.

\section{Finalidade do uso de ferro injetável}
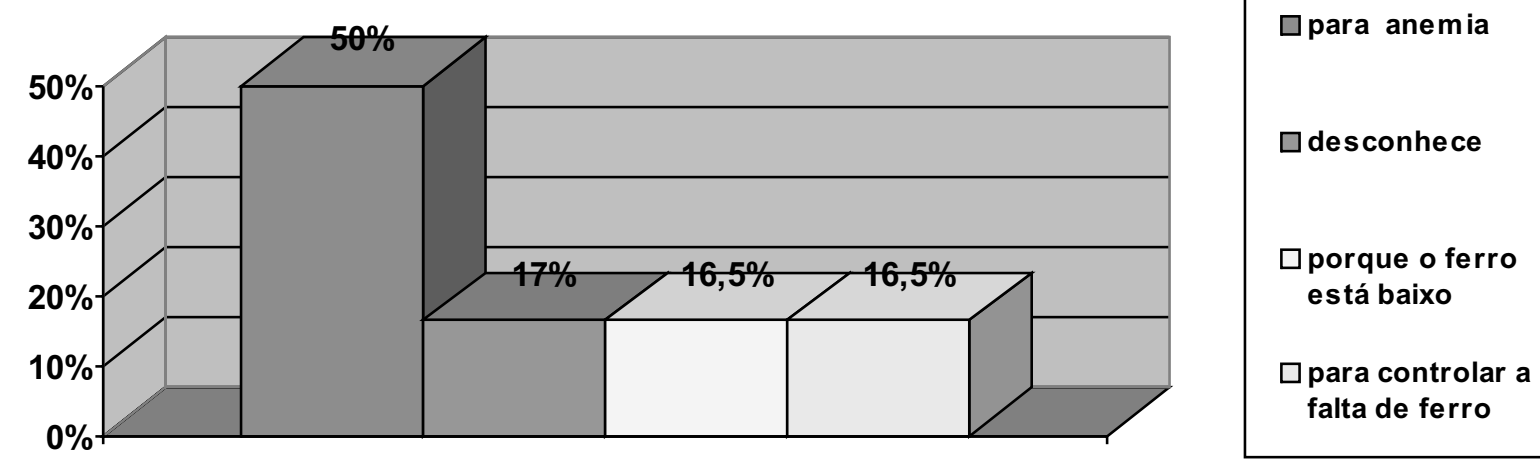

Gráfico 5 - Distribuição dos pacientes (n=11) segundo conhecimento sobre o uso do ferro injetável. Guarapuava, 2002 


\section{CONSIDERAÇÕES FINAIS}

A Insuficiência Renal Crônica é uma enfermidade que, além de trazer conseqüências físicas ao indivíduo que a vivencia, traz prejuízos também de ordem psicológica, emocional e social. A partir da descoberta do diagnóstico da doença renal, estabelecese um longo processo de adaptação a essa nova condição, no qual o indivíduo precisa identificar meios para lidar com o problema renal e com todas as mudanças e limitações que o acompanham.

A necessidade de se adaptar a novas rotinas impostas pelo tratamento se faz necessária e para isso é de extrema importância o apoio da equipe multidisciplinar.

O presente estudo evidenciou as dificuldades e as dúvidas dos pacientes em relação ao seu tratamento e o pouco conhecimento em relação aos suplementos medicamentosos utilizados. A atenção dos profissionais que atuam em nefrologia é crucial, neste sentido, para que os pacientes sejam bem orientados sobre o uso dos medicamentos, contribuindo para a adesão e eficácia.

\section{REFERÊNCIAS}

1. Barros E, Manfro RC, Thomé FS, Gonçalves LFS. Nefrologia: rotinas, diagnóstico e tratamento. Porto Alegre: Artmed; 1999.

2. Martins C, Riella MC. Nutrição e o rim. Rio de Janeiro: Guanabara Koogan; 2001.

3. Lima EB. Opinião do paciente com insuficiência renal crônica, submetido à técnica de auto-administração de medicamentos orais durante a hospitalização [dissertação]. São Paulo (SP): Escola de Enfermagem de São Paulo. Universidade de São Paulo; 1989

4. Castro MC, Caiuby AVS, Draibe SA, Canziani MAF. Qualidade de vida de pacientes com insuficiência renal crônica em hemodiálise avaliada através do instrumento genérico SF-36. Rev Assoc Med Bras. 2003;49(3):245-9.

5. Neves OO, Cruz ICF. Produção científica de enfermagem sobre inserção de cateter endovenoso em fístula arteriovenosa: implicações para a (o) enfermeira (o) de métodos dialíticos [texto na Internet]. [2000?]. [citado 2007 Fev 23] Disponível em: www.uff.br/nepae/ catetervenosoemfistula.doc

6. Braunwald E. Tratado de medicina cardiovascular. $4^{\mathrm{a}}$ ed. São Paulo: Roca; 1996.
7. Giannini SD, Forti N, Diament J. Cardiologia preventiva: prevenção primária e secundária. $5^{\mathrm{a}}$ ed. São Paulo: Atheneu; 2000.

8. Barros E, Thomé F. Prevenção das doenças renais. In: Barros E, Manfro RC, Thomé F, Gonçalves LF, colaboradores. Nefrologia: rotinas, diagnóstico e tratamento. $2^{\mathrm{a}}$ ed. Porto Alegre: Artmed; 1999.

9. Barreto ACP, Santello JL. Manual de hipertensão: entre a evidência e a prática clínica. São Paulo: Lemos Editorial; 2002.

10. Trentini M, Corradi E, Araldi MAR, Tigrinho FC. Qualidade de vida de pessoas dependentes de hemodiálise considerando alguns aspectos físicos, sociais e emocionais. Texto Contexto Enferm. 2004; 13 (1): 74-82.

11. Riella MC. Princípios de nefrologia e distúrbios hidroeletrolítico. Rio de Janeiro: 1988.

12. Daugirdas JT, Ing TS. Manual de diálise. Rio de Janeiro: Medsi; 1996. 